\title{
The lumping of heat transfer parameters in cooled packed beds: effect of the bed entry
}

\author{
E. J. Westerink, J. W. Gerner, S. van der Wal and K. R. Westerterp* \\ Chemical Reaction Engineering Laboratories, Department of Chemical Engineering, Twente University of Technology, PO Box 217 , \\ 7500 AE Enshede (Netherlands)
}

(Received February 27, 1992; in final form September 21, 1992)

\begin{abstract}
The lumping of the heat transfer parameters of the one- and the two-dimensional pseudo-homogeneous model of a cooled fixed bed were compared. It appeared that the lumping of the two-dimensional parameters, being the effective radial conductivity $\lambda_{\mathrm{cff}}$ and the heat transfer coefficient at the wall $\alpha_{\mathrm{w}}$, into the one-dimensional overall heat transfer coefficient $U$ results in a length dependence of $U$. It is shown that the ratio $\alpha_{w} / U$ develops from unity at the bed inlet to a final value. The magnitude of this final value depends on the Biot number, whereas the length of this transition section is affected by the Peclet number. A new relation to lump the effective conductivity and the heat transfer coefficient at the wall into an overall heat transfer coefficient which depends on the values of the Biot number and the Peclet number is presented. This relation accounts for both the magnitude and the transition length of the ratio $x_{w} / U$.
\end{abstract}

\section{Introduction}

For many years investigators have studied heat transfer in packed beds. Several models have been proposed to describe the temperature profiles in cooled packed beds. These models range from a two-dimensional heterogeneous model to a simple one-dimensional pseudohomogeneous model. For all these models the relevant parameters need to be determined from experiments.

In industry usually the pseudo-homogeneous one- or two-dimensional plug flow model is used. For the twodimensional model two parameters are required, being the effective radial heat conductivity inside the bed $\lambda_{\text {eff }}$ and the transfer coefficient at the wall $\alpha_{w}$, whereas for the one-dimensional model only an overall heat transfer coefficient $U$ is needed.

Some authors published correlations for both the effective heat conductivity and the heat transfer coefficient at the wall. Well known are the studies of Dixon and Cresswell [1-3] and of Hennecke, Zehner and Schlünder [4, 5]. Dixon and Cresswell, [2] showed that the heat transfer parameters for the two-dimensional model TDM do not show any bed length effects.

Westerink et al. $[6,7]$ studied parameter regions for which both the one- and the two-dimensional model of the cooled tubular reactor led to the same results in

*Author to whom correspondence should be addressed. view of the mean hot spot temperature, the conversion and the reaction rate. It was found that for the region of the so called low parametric sensitivity both models are equivalent as long as the heat transfer parameters $\lambda_{\text {eff }}$ and $\alpha_{w}$ are properly lumped into one overall heat transfer coefficient $U$. It was shown that the lumping relation proposed by Crider and Foss [8] can be used for this purpose.

A critical analysis of the various experimental methods for the determination of the overall heat transfer coefficient was made by Martinez et al. [9]. They found that lumping via the heterogeneous one-dimensional model leads to better results than in case the Crider and Foss relation is used. They only studied Biot values ranging from $1 \leqslant B i \leqslant 20$.

All relations discussed above are valid for fully developed temperature profiles only. In practice, for a fluid that is cooled down in a packed bed, a radially flat profile is found at the inlet of the bed which then changes throughout the bed until a parabolic shape is reached. $\mathrm{Li}$ and Finlayson [10] presented a criterion to check for what minimum bed length the inlet effects can be neglected.

In this study, these bed entry effects will be discussed and the results into an improved relation for the lumping of $\lambda_{\text {eff }}$ and $\alpha_{w}$ into the overall heat transfer coefficient $U$ will be included.

First, the relevant basic equations will be discussed and how the overall heat transfer parameter $U$ depends 
on the bed length will be shown. The parameter ranges for which the length effect plays an important role will be investigated. Next, an improved lumping relation, including the length dependence of $U$, and a new criterion to check the negligibility of bed inlet effects will be introduced. Finally, how the results compare to the temperature profiles calculated by the two-dimensional model and the one-dimensional model if inlet effects are neglected will be shown. To this end, various laboratory and industrial cases will be discussed.

\section{Basic equations}

For a packed bed; in which a hot fluid is cooled down by a cooling medium at the outside of the tube wall, the heat balance for the two-dimensional plug flow model can be written as:

$\frac{1}{\rho} \frac{\partial}{\partial \rho}\left(\rho \frac{\partial \theta}{\partial \rho}\right)-\mathrm{Pe} \frac{\partial \theta}{\partial \omega}=0$

with boundary conditions:

$\omega=0, \quad 0 \leqslant \rho \leqslant 1, \quad \theta=1$

$\rho=0, \quad 0 \leqslant \omega \leqslant 1, \quad \frac{\partial \theta}{\partial \rho}=0$

$\rho=1, \quad 0 \leqslant \omega \leqslant 1, \quad \frac{\partial 0}{\partial \rho}=-\mathbf{B i} \theta$

These equations are written in a dimensionless form, for the temperature $\theta$ we use $\theta=\left(T(z, r)-T_{\mathrm{c}}\right)$ / $\left(T_{\mathrm{o}}-T_{\mathrm{c}}\right)$, for the axial coordinate $\omega=z / L_{\mathrm{t}}$ and for the radial coordinate $\rho=r / R_{\mathrm{t}}$. Moreover we use two dimensionless groups, being the Biot number $\mathrm{Bi}=\alpha_{\mathrm{w}} R_{\mathrm{t}} /$ $\lambda_{\text {eff }}$ and a modified Peclet number $\mathrm{Pe}=v_{\mathrm{o}} \rho_{\mathrm{g}} C_{\mathrm{pg}} R_{\mathrm{t}}^{2} /$ $\left(L_{\mathrm{l}} \lambda_{\mathrm{efr}}\right)$. Further dispersion of heat in the axial direction has been neglected.

Solving eqn. (1) with the boundary conditions given we obtain:

$\theta=2 \mathrm{Bi} \sum_{i=0}^{\infty} \frac{J_{0}\left(\beta_{i} \rho\right) \exp \left[-\beta_{i}^{2} \omega / \mathrm{Pe}\right]}{\left[\mathrm{Bi}^{2}+\beta_{i}^{2}\right] J\left(\beta_{i}\right)}$

Here the eigenvalues $\beta_{i}$ have to be calculated from: $\operatorname{Bi} J_{0}\left(\beta_{i}\right)=\beta_{i} J_{1}\left(\beta_{i}\right)$. In order to compare the one- and the two-dimensional model we need to calculate the radially averaged temperature in the two-dimensional model, which is

$\bar{\theta}=4 \mathrm{Bi}^{2} \sum_{i=0}^{\infty} \frac{\exp \left[-\beta_{i}^{2} \omega / \mathrm{Pe}\right]}{\left[\mathrm{Bi}^{2}+\beta_{i}^{2}\right] \beta_{i}^{2}}$

The one-dimensional model can be written as:

$-\operatorname{Pe} \frac{\mathrm{d} \bar{\theta}}{\mathrm{d} \omega}=2 \mathrm{Bi}\left[\frac{U}{\alpha_{\mathrm{w}}}\right] \bar{\theta} \quad$ with $\quad \bar{\theta}(\omega=0)=1$
Using eqns. (3) and (4) we can obtain a definition for the overall heat transfer coefficient in relation to the parameter $\alpha_{w}$ of the two-dimensional model:

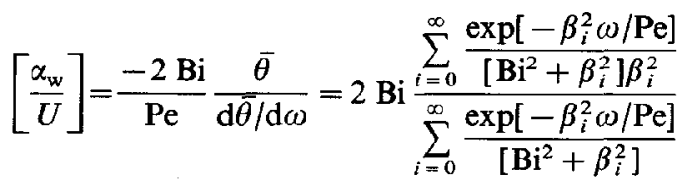

Equation (5) relates the local value of $U$ to the position $\omega$ in the tube. We will use eqns (3)-(5) to study the effect of the tube inlet and transition zone on the lumping of $\alpha_{w}$ and $\lambda_{\text {eff }}$.

\section{Axial development of $\alpha_{w} / U$}

As can be concluded from eqn. (5), the ratio $\alpha_{w} / U$ still depends on the bed length $\omega$. Since both $\alpha_{w}$ and $\lambda_{\text {eff }}$ are true constants, as was shown by Dixon [2], the overall heat transfer coefficient $U$ depends on the bed length. Two limiting cases can be observed, for the bed inlet $\omega=0$ :

$\left[\frac{\alpha_{\mathrm{w}}}{U}\right]_{0}=\frac{2 \mathrm{Bi}}{\operatorname{Pe}} \frac{\bar{\theta}_{\mathrm{o}}}{(\mathrm{d} \bar{\theta} / \mathrm{d} \omega)_{\mathrm{o}}}=1$

and for sufficiently large values of $\omega$ where only the first term of the summations need to be evaluated, which gives us:

$\lim _{\omega \rightarrow \infty}\left[\frac{\alpha_{\mathrm{w}}}{U}\right]_{\infty}^{2}=\frac{2 \mathrm{Bi}}{\beta_{0}^{2}}$

In Fig. 1 we plotted the value of $\alpha_{w} / U$ as a function of the axial position $\omega$ for several values of the Peclet number Pe. As can be seen from Fig. 1 for high values of the Peclet number the profile develops slowly. In Fig. 2 we plotted the ratio $\alpha_{w} / U$ for several values of the Biot number. From this figure we can see that large differences between the tube inlet and the fully developed profile occur for large values of the Biot number,

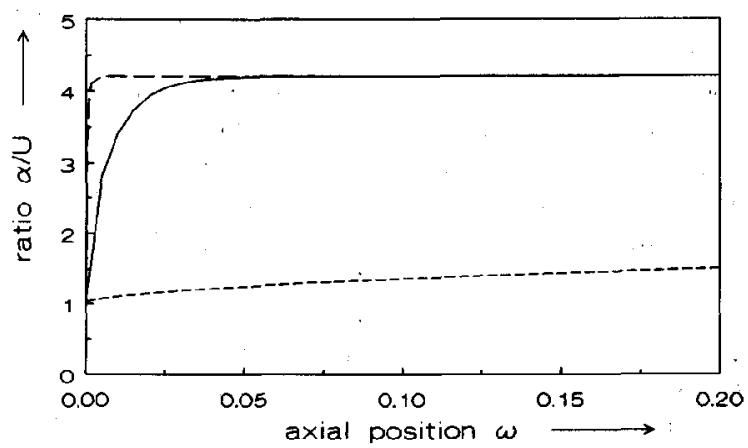

Fig. 1. Development of the ratio $\alpha_{w w} / U$ as a function of the axial coordinate $\omega$ for several values of the Peclet number. Here $\mathrm{Bi}=10$ and $\mathrm{Pe}=0.01,(---) ; 0.2,(-)$ and $100,(--)$. 


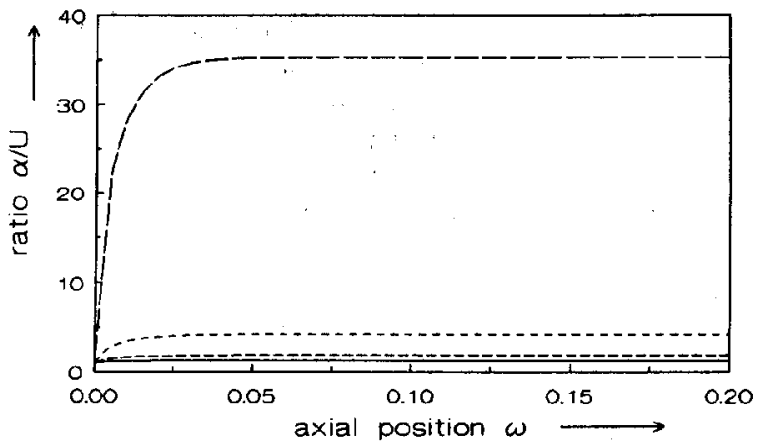

Fig. 2. Development of the ratio $\alpha_{\mathrm{w}} / U$ as a function of the axial coordinate $\omega$ for several values of the Biot number. Here $\mathrm{Pe}=0.2$ and $\mathrm{Bi}=1,(-) ; 3,(---) ; 10,(--)$ and $100,(---)$.

this is the case for fixed bed reactors with large $D_{\mathrm{t}} / D_{\mathrm{p}}$ ratios or a large number of particles on a tube diameter and with low fluid velocities.

\section{Fully developed profiles}

Firstly the relations for fully developed profiles will be studied and so for $\left(\alpha_{w} / U\right)_{\omega \rightarrow \infty}$, the analytical solution was given by eqn. (7). In this relation the value of $\beta_{n}^{2}$ is a function of the Biot number via the eigenvalue relation in eqn. (1). It can be correlated in various ways, for example:

Beek [11]: $\left[\frac{\alpha_{\mathrm{w}}}{U}\right]_{\omega \rightarrow \infty}=1+\mathrm{Bi} / 4 \quad 0<\mathrm{Bi}<1$

Crider and Foss [8]:

$\left[\frac{\alpha_{\mathrm{w}}}{U}\right]_{\omega \rightarrow \infty}=1+\mathrm{Bi} / 3.06 \quad 1 \leqslant \mathrm{Bi} \leqslant 50$

Limiting case:

$\lim _{B i \rightarrow \infty} \beta_{o}^{2}=5.78\left[\frac{\alpha_{w}}{U}\right]_{\omega \rightarrow \infty}=B i / 2.89 \quad \mathrm{Bi}>50$

see Crider and Foss [8]

All these relations are applicable in a specific region of Biot values. We have re-evaluated the eigenvalue relation and propose the following relation for $\left(\alpha_{w} / U\right)_{w \rightarrow \infty}$ for the entire region $0 \leqslant B i \leqslant \infty$ :

$\left[\frac{\alpha_{w}}{U}\right]_{\omega \rightarrow \infty}=1+\frac{\mathrm{Bi}}{2.89+1.11 /(1+\mathrm{Bi})^{0.68}}$

The quality of this fit was compared with the relations given by eqns. (8) $-(10)$. The result is shown in Fig. 3, where the error in $\left(\alpha_{\mathrm{w}} / U\right)_{\omega \rightarrow \infty}$ is plotted as a function of the Biot number. The error is defined as, see eqn. (7):

error $=\frac{2 \mathrm{Bi} / \beta_{\mathrm{o}}^{2}-\mathrm{Fit}}{2 \mathrm{Bi} / \beta_{\mathrm{o}}^{2}}$

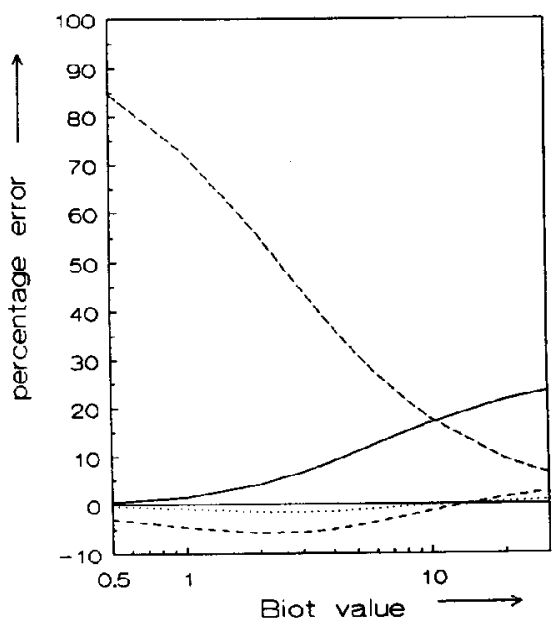

Fig. 3. The error in $\left(\alpha_{w} / U\right)_{\omega \approx \infty}$ as a function of the Biot number for the relations of Beek [11], ( $\longrightarrow$ ); Crider and Foss [8], (- -); for $\mathrm{Bi} / 2.89,(-\ldots-)$ and for this work $(\cdots)$.

From this figure we may conclude that our relation leads to maximum errors less than $2 \%$ whereas the other relations have maximum errors from $-6 \%$ for Crider and Foss [8] to $100 \%$ in the limiting case $\mathrm{Bi} \rightarrow 0$. From now on eqn. (11) for the estimation of the value of $\alpha_{w} / U$ for fully developed profiles, will be used.

Effect of the Peclet number on $\alpha_{\mathrm{w}} / U$

As stated before the ratio $\alpha_{w} / U$ develops from unity at the tube inlet to $2 \mathrm{Bi} / \beta_{\mathrm{o}}^{2}$ at some location along the bed axis. This can be a point beyond the end of the bed. In order to study the development of the ratio $\alpha_{w} / U$ we plotted values of $\left(\alpha_{w} / U\right)_{\omega-1}$ as a function of the Biot number. This is shown in Fig. 4 and it appeared that for large Biot values the lines become straight. For small values of the Peclet number the ratio $\alpha_{\mathrm{w}} / U$ develops so fast that at $\omega=1$ the final value $2 \mathrm{Bi} / \beta_{\text {o }}^{2}$ is already reached, which is equal to $\mathrm{Bi} / 2.89$ for large Biot values. Hence, the maximum possible tangent of lines of $\alpha_{\mathrm{w}} / U$ versus $\mathrm{Bi}$ is $1 / 2.89$. Using the results from Fig. 4 the effect of the Peclet number was incorporated on the ratio $\alpha_{w} / U$ by fitting $\mathrm{f}(\mathrm{Pe})$ in the following relation:

$\left(\alpha_{\mathrm{w}} / U\right)_{\omega-1}=1+\mathrm{f}(\mathrm{Pe}) / 2.89$

to the tangents found in Fig. 4. It was found that the relation:

$\mathrm{f}(\mathrm{Pe})=1-\exp \left(-8.5 / \mathrm{Pe}^{0.58}\right)$

fits very well. Therefore the following relation for $\left(\alpha_{w} / U\right)_{\omega=1}$ in case any axial dependence should be taken into account is suggested:

$\left[\frac{\alpha_{\mathrm{w}}}{U}\right]_{\omega=1}=1+\frac{1-\exp \left(\frac{-8.5}{\mathrm{Pe}^{0.58}}\right)}{2.89+\left\{1.11 /(1+\mathrm{Bi})^{0.68}\right\}} \times \mathrm{Bi}$ 


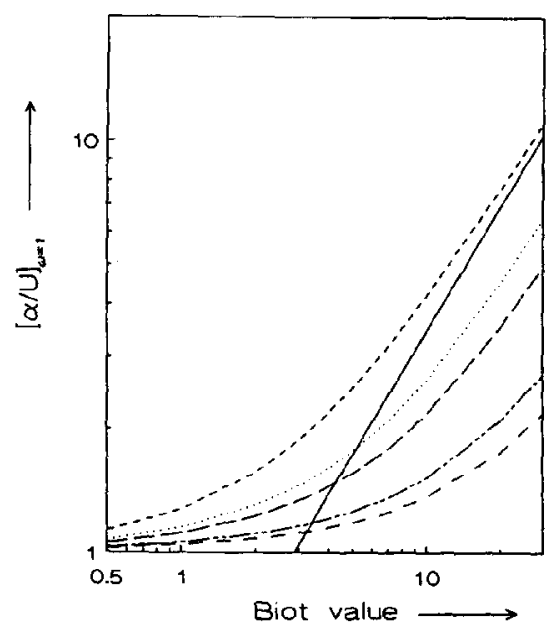

Fig. 4. Development of $\left(\alpha_{w} / U\right)_{w \approx 1}$ as a function of the value of the Biot number. The lines are for the following Peclet values $0.01,(--)$; 1, (- -); 50, ( . ) ; 100, ( ---$)$; 500, ( - - $)$ and $1000,(---)$. The lines for $\mathrm{Pe}=0.01$ and 1 coincide. Also the relation $\left(\alpha_{\mathrm{w}} / U\right)_{\infty}=\mathrm{Bi} / 2.89(-)$ is shown.

or after we introduce the axial coordinate $z$ :

$$
\left[\frac{\alpha_{\mathrm{w}}}{U}\right]_{\mathrm{z}}=1+\frac{1-\exp \left(-8.5\left[\frac{z}{\operatorname{Pe} L_{\mathrm{t}}}\right]^{0.58}\right)}{2.89+\left\{1.11 /(1+\mathrm{Bi})^{0.68}\right\}} \times \mathrm{Bi}
$$

The relation given by eqn. (13) describes the ratio between the overall resistance towards heat transfer and the resistance to heat transfer at the wall at any axial position $z$.

\section{A criterion for axial development}

For any design engineer involved in heat transfer problems in packed beds it is important to know whether the resistance to heat transfer is a strong function of the axial position in case he uses a one-dimensional model. From eqn. (13) a useful criterion can be obtained if we define our criterion as:

$\frac{\left(\alpha_{\mathrm{w}} / U\right)_{\omega \rightarrow \infty}-\left(\alpha_{\mathrm{w}} / U\right)_{\omega}}{\left(\alpha_{\mathrm{w}} / U\right)_{\omega \rightarrow \infty}-\left(\alpha_{\mathrm{w}} / U\right)_{\omega=0}}<0.05$

which means that at a position $\omega$ the difference between the local heat transfer coefficient and its final value at $\omega=\infty$ is less than $5 \%$ of its maximum value. This is a conservative criterion since the difference between initial value and final value may be small as is the case for small Biot values. After substitution, where $\left(\alpha_{w} / U\right)_{\omega=0}=1$, we get:

$\omega_{\text {min }} \geqslant \operatorname{Pe}\left[\frac{L_{\mathrm{t}}(1 / 0.05)}{8.5}\right]^{1 / 0.58}>0.17 \mathrm{Pe}$
So for low Pe numbers the transition zone is short, its length is $z_{\text {trans }}=0.17 L_{\mathrm{t}} \mathrm{Pe}$. It should be realised that our modified Peclet number is equivalent to $\mathrm{Bo}_{\mathrm{rh}}\left(d_{\mathrm{t}} / d_{\mathrm{p}}\right)$ / $\left(4 L_{1} / d_{t}\right)$. The Bodenstein number $\mathrm{Bo}_{\mathrm{rh}}$ for radial transport of heat varies between 8-16 and further the number of particles on a tube diameter $d_{\mathrm{t}} / d_{\mathrm{p}}$ as well as the tube slenderness $L_{\mathrm{t}} / d_{\mathrm{t}}$ are known, so eqn. (14) enables us to estimate quickly the length of the transition zone in which the $\alpha_{w} / U$ profiles develop.

\section{Comparison of temperature profiles}

Until now we have only studied the ratio $\alpha_{w} / U$ as a function of the tube length, in practice we are interested in the actual temperature profile taking into account the length dependence of $U$.

To illustrate this effect the temperature profiles for three combinations of the Peclet number and the Biot number have been plotted. These profiles are shown in Figs. 5, 6 and 7. In these figures four lines have been plotted, the first line corresponds to the analytical solution of the temperature profile as calculated by the

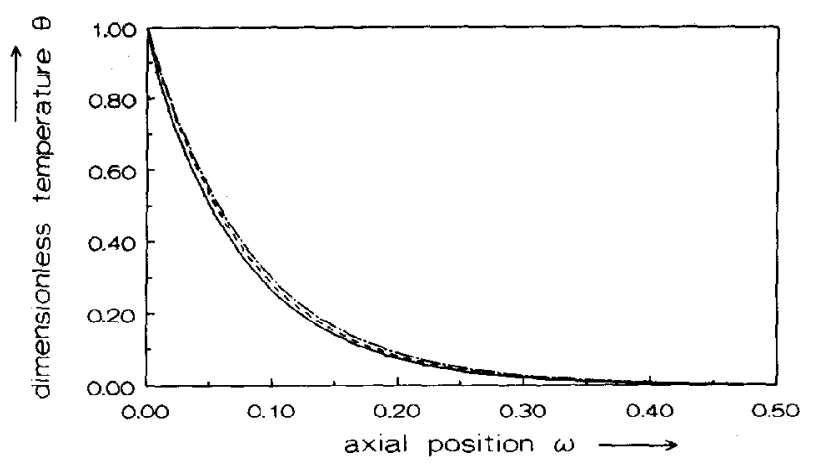

Fig. 5. Comparison of the temperature profiles calculated from the exact solution $\left(\longrightarrow\right.$ ), the length independent values of $\alpha_{w} / U$ given by Crider and Foss [8], (- - - ), and Westerink (-- $)$ and the axial dependent value of $\alpha_{w} / U$ as given by eqn. (12) $(\cdots)$. Here $\mathrm{Pe}=1$ and $\mathbf{B i}=5$.

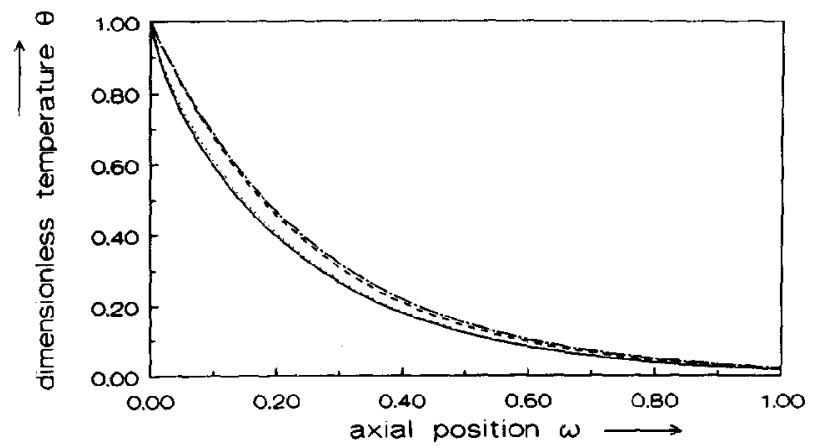

Fig. 6. Same as Fig. 5, except here $\mathrm{Pe}=1$ and $\mathrm{Bi}=5$. 


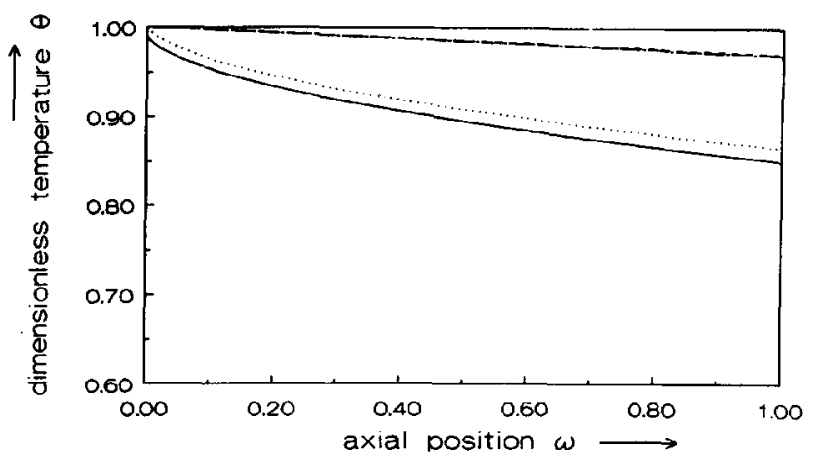

Fig. 7. Same as Fig. 5, except here $\mathrm{Pe}=200$ and $\mathbf{B i}=100$.

two-dimensional model eqn. (3), the second line corresponds to integration of eqn. (4) with a constant value of $\alpha_{w} / U$ as defined by our relation eqn. (11). The third line shows the effect of the length dependence of $\alpha_{w} / U$, since here relation (13) is used in combination with the integration of eqn. (4). Finally the temperature profile was plotted in case the relation of Crider and Foss [8] eqn. (9) is used if eqn. (4) is integrated.

In Fig. 5 the temperature profile for $\mathrm{Pe}=0.2$ and $\mathrm{Bi}=2$ has been plotted, this corrresponds to a packed hed with a small tube to particle diameter ratio, and a good effective conductivity inside the bed. This situation is achieved in a cooled tubular reactor which is operated at high gas loads. It can be seen that there is hardly any effect of the length dependence of $U$. The final value of $U$ is reached shortly after the tube inlet.

In Fig. 6, where $\mathbf{P e}=1$ and $\mathbf{B i}=5$, it can be seen that for small values of the Peclet number and large values of the Biot number the profile is not much affected by the length dependence of $U$. The values of $\mathrm{Bi}$ and $\mathrm{Pe}$ chosen, refer to a short, thick laboratory tube at low flows. The low value of the Peclet number causes a rapid development of $\alpha_{w} / U$. However, it is more the quality of the fit of $\left(\alpha_{\mathrm{w}} / U\right)_{\mathrm{Bi} \rightarrow \infty}$ that determines the error between the actual and the predicted value of the temperature.

Finally in Fig. 7, what happens if the ratio $\alpha_{\mathrm{w}} / U$ develops only slowly, can be seen, here $\mathrm{Pe}=200$ and $\mathbf{B i}=100$. The values chosen for $\mathrm{Pe}$ and $\mathrm{Bi}$ typically correspond to an adiabatic bed reactor with a high Reynolds number. It can be seen that both relations with a constant value of $\alpha_{\mathrm{w}} / U$ predict a much slower decrease in temperature than the two lines that account for the length dependence. Also it is shown that our eqn. (13) predicts the development of $\alpha_{w} / U$ well.

\section{Total heat supply or heat loss}

To estimate the total heat supply $\bar{U}$ the overall heat transfer coefficient $U$ over the length $\omega$ has to be integrated:
$\bar{U}=\int_{0}^{1} U(\omega) \mathrm{d} \omega$

where $U(\omega)$ can be obtained from eqn. (13). This equation does not have an analytical solution. However, for values of $\mathrm{Pe}$ smaller than 1 , that is, of cooled packed bed reactors, the influence of $\mathrm{Pe}$ is negligible. In this case the following relation for $\bar{U}$ is obtained:

$$
\bar{U}=\frac{\alpha_{\mathrm{w}}}{1+\mathrm{Bi} /\left[2.89+\left\{1.11 /(1+\mathrm{Bi})^{0.68}\right\}\right]} \quad \mathrm{Pe} \leqslant 1
$$

For higher Pe values a numerical approximation of the total heat supply has to be made.

\section{Conclusions}

In this paper the lumping of the two-dimensional parameters $\lambda_{\text {eff }}$ and $\alpha_{\mathrm{w}}$ into the one-dimensional model parameter $U$ has been studied. The region of the tube inlet was the subject of particular interest. It was shown that for high Peclet numbers the value of $U$ varies along the tube axis. This phenomenon can have a strong effect on the temperature profile that is predicted by the one-dimensional model.

In the case of long industrial reactors, where the values both of the Peclet number and the Biot number are small (e.g. $P e=0.2$ and $B i=2$ ), it was shown that the lumping of $\alpha_{w}$ and $\lambda_{\text {eff }}$ leads to more or less the same results regardless of the lumping relation that is used, either our new relation or the Crider and Foss [8] relation can be used in the one-dimensional model. In this situation the gas is usually already at the same temperature as the coolant, therefore no problems will arise.

For higher values of the Peclet and Biot number (e.g. $\mathrm{Pe}=1$ and $\mathrm{Bi}=5$ ) which are achieved in short, thick laboratory reactors the lumping with a length dependent relation for $U$, eqn. (13), gives the best results.

Finally, for large values of both the Peclet number and the Biot number (e.g. $\mathrm{Pe}=200$ and $\mathrm{Bi}=100$ ) the use of either the two-dimensional model or the one-dimensional model with a length dependent value of $U$, eqn. (13), is strongly recommended. This situation is achieved in adiabatic bed reactors. In any case, the use of the criterion

$\left(\frac{L_{\mathrm{t}} \hat{\lambda}_{\mathrm{eff}}}{G_{\mathrm{mass}} C_{\mathrm{pg}}}\right)_{\min } \geqslant 0.054$

to check if $U$ can be considered constant along the tube axis is advised. 


\section{Nomenclature}

$\mathrm{Bi} \quad=\alpha_{\mathrm{w}} R_{\mathrm{t}} / \lambda_{\text {eff }}$, Biot number

Bo $\quad=\frac{\rho_{\mathrm{g}} C_{\mathrm{pg}} v_{\mathrm{o}} d_{\mathrm{p}}}{\lambda_{\mathrm{eff}}}$, Bodenstein number

$C_{\mathrm{pg}} \quad$ specific heat of fluid, $\mathrm{J} \mathrm{kg}^{-1} \mathrm{~K}^{-1}$

$d_{\mathrm{p}} \quad$ particle diameter, $\mathrm{m}$

$d_{\mathrm{t}} \quad$ tube diameter, $\mathrm{m}$

$G_{\text {mass }}$ mass flow of fluid, $\mathrm{kg} \mathrm{s}^{-1}$

$J_{0}, J_{1}$ Bessel functions of zero and first order

$L_{\mathrm{t}} \quad$ tube length, $\mathrm{m}$

Pe $\quad=v_{\mathrm{o}} \rho_{\mathrm{g}} C_{\mathrm{pg}} R_{\mathrm{t}}^{2} /\left(L_{\mathrm{t}} \lambda_{\mathrm{cff}}\right)$, Peclet number

$r \quad$ radial coordinate, $\mathrm{m}$

$R_{\mathrm{t}} \quad$ tube radius, $\mathrm{m}$

$T \quad$ temperature, $\mathrm{K}$

$U$ overall heat transfer coefficient, $\mathrm{W} \mathrm{m}^{-2} \mathrm{~K}^{-1}$

$\bar{U} \quad$ total heat supply/loss, $\mathrm{W} \mathrm{m}^{-2} \mathrm{~K}^{-1}$

$v_{0} \quad$ superficial fluid vclocity, $\mathrm{m} \mathrm{s}^{-1}$

$z \quad$ axial coordinate, $\mathrm{m}$

\section{Greek letters}

$\alpha_{\mathrm{wv}} \quad$ wall heat transfer coefficient, $\mathrm{W} \mathrm{m} \mathrm{m}^{-2} \mathrm{~K}^{-1}$

$\beta_{i} \quad i^{\text {th }}$ eigenvalue of relation in eqn. (2)

$\theta \quad=\left(T-T_{\mathrm{c}}\right) /\left(T_{\mathrm{o}}-T_{\mathrm{c}}\right)$ dimensionless temperature

$\bar{\theta} \quad$ dimensionless mean radial temperature

$\lambda_{\text {eff }} \quad$ effective radial conductivity, $\mathrm{W} \mathrm{m}^{-1} \mathrm{~K}^{-1}$

$\rho \quad=r / R_{\mathrm{t}}$ dimensionless radial coordinate

$\rho_{\mathrm{g}} \quad$ fluid density, $\mathrm{kg} \mathrm{m}^{-3}$

$\omega \quad=z / L_{\mathrm{t}}$ dimensionless axial coordinate

\section{Subscripts}

c at coolant conditions o at inlet conditions

rh in the radial direction for heat

TDM for the two-dimensional model

\section{References}

1 A. G. Dixon and D. L. Cresswell, Theoretical prediction of effective heat transfer parameters in packed beds, AIChE J., 25 (1979) 663-676.

2 A. G. Dixon and D. L. Cresswell, The length effect on packed bed effective heat transfer parameters, Chem. Eng. J., 31 (1985) 163-173.

3 A. G. Dixon, Effective heat transfer parameters for transient packed bed models, AIChE J., 32 (1986) 809-819.

4 F. W. Hennecke and E. U. Schlünder, Wärmeübergang in beheizten oder gekühlten Rohren mit Schüttungen aus Kugeln, Zylindern und Raschig-Ringen, Chem.-Ing.-Tech., 45 (1973) $277-284$

5 P. Lehner and E. U. Schlünder, Die effektive Wärmeleitfähigkeit durchströmter Kugelschüttungen bei mäßigen und hohen Temperaturen, Chem.-Ing.-Tech., 45 (1973) 272-276.

6 E. J. Westerink, Heat effects in chemical reactors. Design and Stability, $P h D$ thesis, Twente University of Technology, 1988.

7 E. J. Westerink, N. Koster and K. R. Westerterp, The choice between cooled tubular reactor models: analysis of the hot spot, Chem. Eng.. Sci., 45 (1990) 3443-3455.

8 J. E. Crider and A. S. Foss, Effective wall heat transfer coefficients and thermal resistances in mathematical models of packed beds, AIChE J., 11 (1965) 1012-1019.

9 O. M. Martínez, S. I. Pereira Duarte, O. A. Ferretti and N. O. Lemcoff, Estimation of the pseudo homogeneous one-dimensional heat transfer coefficient in a fixed bed, Chem. Eng. Process., 20 (1986) 245-253.

10 C. H. Li and B. A. Finlayson, Heat transfer in packed beds. A reevaluation, Chem. Eng. Sci., 32 (1977) 1055-1066.

$11 \mathrm{~J}$. Beek, Design of packed catalytic reactors, in $\mathrm{Th}$. Drew, J. W. Hoopes and Th. Vermeulen (eds.), Advances in Chemical Engineering, Academic Press, New York, 1962, Vol. 3, pp. 203-271. 